\title{
Complete response to avelumab and IL- 15 superagonist N-803 with Abraxane in Merkel cell carcinoma: a case study
}

\author{
Leylah Drusbosky, ${ }^{1}$ Chaitali Nangia, ${ }^{2}$ Andrew Nguyen, ${ }^{1,3}$ Christopher Szeto, ${ }^{1,3}$ \\ Yulia Newton, ${ }^{1,3}$ Patricia Spilman, ${ }^{4}$ Sandeep Bobby Reddy ${ }^{1,3}$
}

To cite: Drusbosky L, Nangia C, Nguyen A, et al. Complete response to avelumab and IL-15 superagonist $\mathrm{N}-803$ with Abraxane in Merkel cell carcinoma: a case study. Journal for ImmunoTherapy of Cancer 2020;8:e001098. doi:10.1136/ jitc-2020-001098

- Additional material is published online only. To view please visit the journal online (http://dx.doi.org/10.1136/jitc2020-001098).

Accepted 10 July 2020

\section{ABSTRACT}

Merkel cell carcinoma (MCC) is a rare aggressive form of skin cancer originating in neuroendocrine cells. The antiprogrammed death ligand 1 (PD-L1) monoclonal antibody (mAb) avelumab has been approved for treatment of MCC, but options are limited, should it be ineffective as a monotherapy. Combined therapy with low/moderate dose nab-paclitaxel and an interleukin 15 (IL-15)-based therapeutic such as the IL-15 'superagonist' N-803 may increase response by activation of the immune system. The case of a 71-year-old man diagnosed with MCC who achieved and maintained a complete response (CR) by treatment with the anti-PD-L1 mAb avelumab in combination with IL-15 superagonist N-803 and nabpaclitaxel (Abraxane) is presented. Avelumab treatment alone resulted in a response in a para-aortic lesion, but not the other tumor masses. N-803 was added, followed by nab-paclitaxel; CT showed a decrease in the size of the abdominal mass at 1 month, near resolution at 3 months and $\mathrm{CR}$ at 5 months. Abraxane was discontinued after the first $\mathrm{CR}$ on $\mathrm{CT}$, and the patient continues on avelumab/N-803 treatment and maintains a CR. Combination of avelumab with low/moderate-dose chemotherapy and an immune enhancer such as N-803 may offer a viable treatment option for MCC patients for whom avelumab therapy alone was not effective.

\section{INTRODUCTION}

Merkel cell carcinoma (MCC) is a rare, aggressive cancer of the skin, originating in neuroendocrine (NE) cells and associated with Merkel cell polyoma virus (MCPyV) in $50 \%-80 \%$ of cases and UV-induced mutagenesis in the remaining cases, with some exceptions. ${ }^{12}$ When present, expression of MCPyV $\mathrm{T}$ antigens is required for MCC tumor cell survival, suggesting it is a target for immunotherapy. ${ }^{3}$ UV-induced MCC is associated with high tumor mutational burden (TMB), ${ }^{2}$ a finding that also supports the use of immunotherapy. MCC treatment consists of surgery, adjuvant radiotherapy and/or immune checkpoint inhibitor therapy in metastatic disease. $^{45}$

Avelumab, an antiprogrammed death ligand 1 (PD-L1) monoclonal antibody, was
FDA approved for use in MCC in March of 2017 (it is also approved in the EU and Japan) based on data from the JAVELIN Merkel 200 trial in which the overall response rate was $33 \% .^{67}$ Responses were observed early and duration ranged from 2.8 to $23.3+$ months with $86 \%$ of responses being durable for 6 months or longer. Responses were observed in patients regardless of PD-L1 tumor expression or presence of $\mathrm{MCPyV}^{8}$ Avelumab is thus the first therapeutic agent specifically approved for use in this indication, independent of line of treatment.

The interleukin-15 (IL-15) superagonist $\mathrm{N}-803$ (also known as ALT-803) ${ }^{9}$ is an experimental compound comprizing a human IL-15 variant bound to a dimeric human IL-15 receptor $\alpha$ sushi domain/human $\mathrm{IgG}_{1}$ Fc fusion protein that acts as a growth and activation factor for natural killer (NK) cells as well as effector and memory $\mathrm{T}$ cells targeting both the innate and adaptive immune systems. ${ }^{1011} \mathrm{~N}-803$ alone or combination with an anti-PD-L1 antibody has been shown to elicit robust antitumor immune responses and prolonged survival in tumorbearing mice. ${ }^{1213} \mathrm{~N}-803$ has also been shown to increase PD-L1 expression both in vitro ${ }^{14}$ and in breast-tumor-bearing mice ${ }^{15}$; and it has been suggested that this may allow lymphocytes to become targets of anti-PD-L1 therapy.

Current data support the use of combination immunotherapy of N-803 with PD-1/ PD-L1 inhibitors. Encouraging phase $1 \mathrm{~b} / 2$ clinical trial data indicate the combination of anti-PD-1 nivolumab and N-803 may be effective in treating checkpoint inhibitor-relapsed patients by enhancing NK and T-cell attack while checkpoint inhibitors prevent exhaustion. ${ }^{16}$ Several clinical trials are underway to determine efficacy of N-803 in combination with haNK (genetically engineered with the high binding affinity Fc receptor FcyRlll-aNK cells; NCT02465957), immunotherapy 




Figure 1 Patienthistory timeline. The patient's cancer, first localized to the gastroesophageal (GE) junction, and was characterized as small cell. He received carboplatin, etoposide and radiation (XRT); the disease was initially stable with the emergence of adenopathy and a PAL mass. Approximately 1.5 years after initial diagnosis, the patient underwent abdominal wall mass resection. Pathology and IHC suggested MCC. This surgery was shortly followed by a second excision of the abdominal wall and partial omenectomy. The patient then received carboplatin and etoposide (carbo/etopo) for an additional 3 months. This resulted in stable disease (SD) for just less than a year, after which CT/PET revealed multiple hepatic lesions, a persistent PA mass, adenopathy and a pelvic mass (Jan 2018 CT). A core needle biopsy (CNB) was performed and treatment with Avelumab (Bevencio) commenced and continued for 2 months before the emergence of diverticulitis (June 2018 CT). After resolution of the diverticulitis by treatment, Avelumab was resumed with addition of N-803 in August 2018. A CT in October 2018 suggested possible bone metastasis (although no progression in abdominal mass), therefore, Abraxane was added in November 2018; and the subsequent December 2018 CT showed a reduction in the abdominal mass. Continued combined treatment resulted in near-complete resolution of the abdominal mass by March 2019. By May 2019, there was no hypermetabolism seen and this complete response (CR) was confirmed in September 2019 (Sep 2019 CT) and to the time this manuscript was written. IHC, immunohistochemistry; MCC, Merkel cell carcinoma; PET, positron emission tomograph.

(NCT03228667) and orchestrated, multimodel vaccine protocols (NCT03167164).

Abraxane-paclitaxel bound to albumin shown to have increased efficacy-is in widespread use for non-small cell lung cancer (NSCLC), breast cancer and pancreatic cancer; and is being studied in other cancers. ${ }^{17-19}$ Recently, combined use of Abraxane with anti-PD-L1 therapies such as atezolizumab has achieved notable results, including prolonged progression-free survival among patients with metastatic triple-negative breast cancer $(\mathrm{mTNBC})^{20}$ and similar findings for NSCLC have been reported. ${ }^{21}$ In addition to additive effects of combined therapy, it has also been described that the release of tumor cell-associated antigens (TAAs) by low-dose or moderate-dose Abraxane can work synergistically with immunotherapy to elicit a vaccine-like antitumor response $\mathrm{e}^{22}$ and, furthermore, that taxanes such as Abraxane can act as lipopolysaccharide mimetics and activate macrophages to direct tumor cell elimination. ${ }^{23}$

\section{CASE REPORT}

We present here a case report on a 71-year-old man diagnosed with a history of gastroesophageal junction poorly differentiated carcinoma histologically appearing as small cell treated (without resection) initially with carboplatin/ etoposide/XRT followed by four more cycles of carboplatin/etoposide (figure 1) ending February 2015. He achieved a mixed response, with residual disease resected from the abdominal wall and new pathology consistent with MCC (table 1). Treatment postresection consisted of 3 months of carboplatin/etoposide resulting in stable disease for approximately 20 months. On July 2016, the patient underwent abdominal surgery and wide excision of the MCC. Surgical margins were negative.

By April 2018, progressive disease was seen. On progression, a core needle biopsy (CNB) of the progressed tumor showed stage IV metastatic MCC (figure 1, January 2018 CT) and in May 2018 avelumab was initiated. After two courses of avelumab, the patient experienced acute diverticulitis. Imaging at that time showed response of the paraaortic lesion (figure 1, June 2018 CT) but not the other tumor masses. Avelumab was discontinued and symptoms resolved after a course of levofloxacin and metronidazole. Avelumab was restarted and, because CT imaging did not show a satisfactory response, after one cycle N-803 was 
Table 1 IHC of primary and CNB tumor tissue

\begin{tabular}{ll}
\hline Antigen & Labeling (NEG/POS) \\
\hline Primary & NEG \\
CK7 & POS \\
CK20 & NEG \\
CD56 & NEG \\
Chromogranin & Weak POS \\
Synaptophysin (Syp) & NEG \\
TTF-1 & \\
\hline CNB & NEG \\
CK7 & POS \\
CK20 & Neg \\
CD56 & POS \\
CD99 & NEG \\
Chromogranin & POS \\
FLI-1 & Weak POS \\
Synaptophysin (Syp) & NEG \\
\hline TdT & NEG \\
TTF-1 & Strong POS \\
AE1/AE3 & \\
\hline
\end{tabular}

CNB, core needle biopsy; IHC, immunohistochemistry; NEG, negative; POS, positive. added. An initial CT performed approximately 2 months after combined avelumab/N-803 therapy suggested possible bone metastasis and increasing activity of the target lesion, therefore, Abraxane $\left(100 \mathrm{mg} / \mathrm{M}^{2}\right.$; at days 1 , 8 and 15 every 28 days) was added to further stimulate the immune response to the tumor(s). One month later, CT showed a decrease in size of the abdominal mass and, with continued avelumab/N-803/Abraxane treatment, near resolution of the mass was seen within 3 months and complete response (CR) at 5 months; continued CR for the para-aortic mass at that time is shown in figure 1, September 2019 CT. Abraxane was discontinued after the first CR on CT, the patient continues on avelumab/N-803 treatment and has maintained a CR for more than 12 months at the time of this report.

\section{Morphology and biomarker labeling in CNB}

MCC is a NE carcinoma and, uncommonly, squamous or sarcomatoid differentiation may be seen. Here, a differential diagnosis of SCLC vs MCC was under consideration because the patient had emphysema, although he described himself as a non-smoker, and there was no cutaneous primary. Immunohistochemical analysis of the tumor typically shows characteristic perinuclear CK20 staining, found in this case both in the resected tumor and the CNB (table 1); CK20 is negative in SCLC. TTF-1 (thyroid transcription factor 1) was negative in

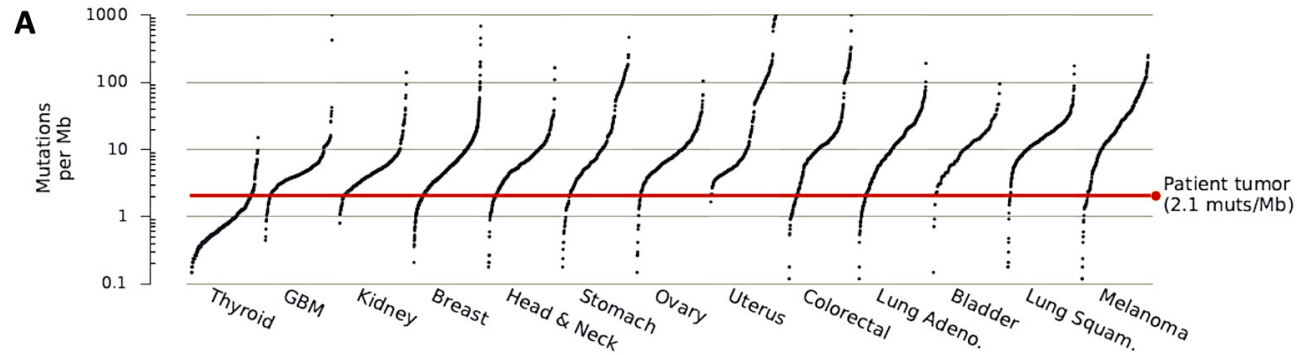

B

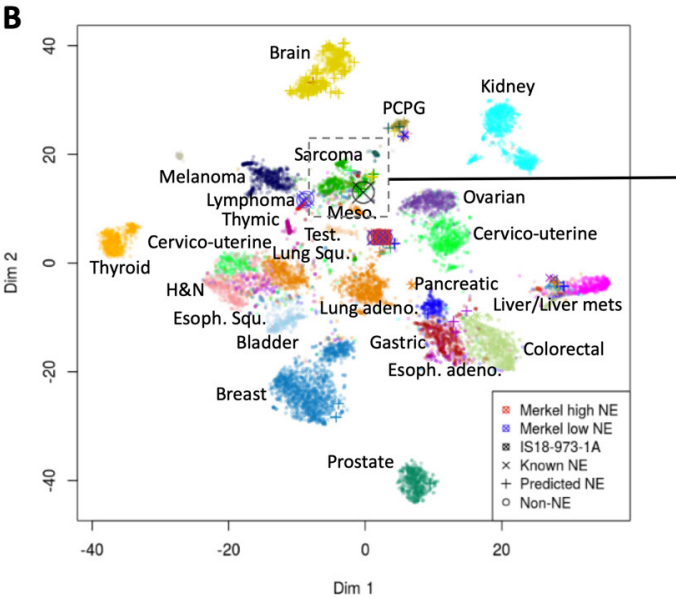

C

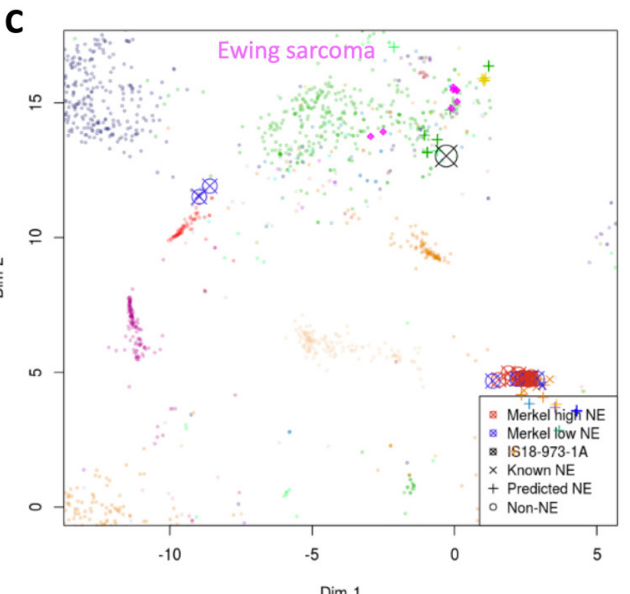

Figure 2 TMB and T-Distributed Stochastic Neighbor Embedding plot for the MCC CNB. (A) The exonic mutation rate for the CNB of the tumor as compared with 5013 other tumor samples is shown as a red line. A total of 446 somatic variants were identified in the patient's CNB, including 44 non-synonymous variants, for an estimated rate of 2.1 mutations per megabase. The character of the CNB in this case was visualized in relationship to a background cohort of $>32$ tumor types, including known and predicted NE and MCC cases, based on the top 3000 most varying genes in this cohort. (B) The tumor (X) clustered with sarcomas and some NE tumors, although not with the main NE cluster (C). CNB, core needle biopsy; MCC, Merkel cell carcinoma; NE, neuroendocrine; TMB, tumor mutational burden. 

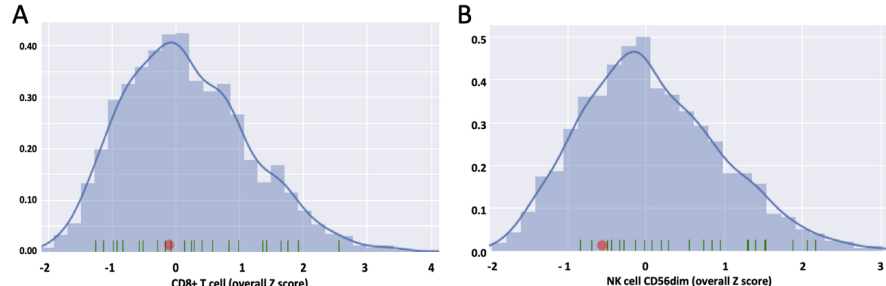

E



H

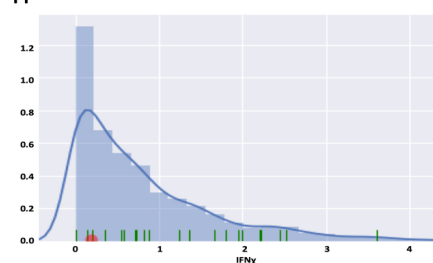

F

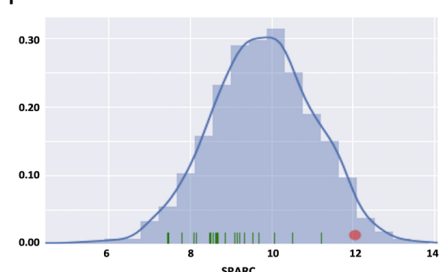

I

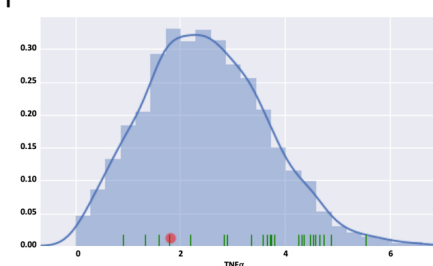

C
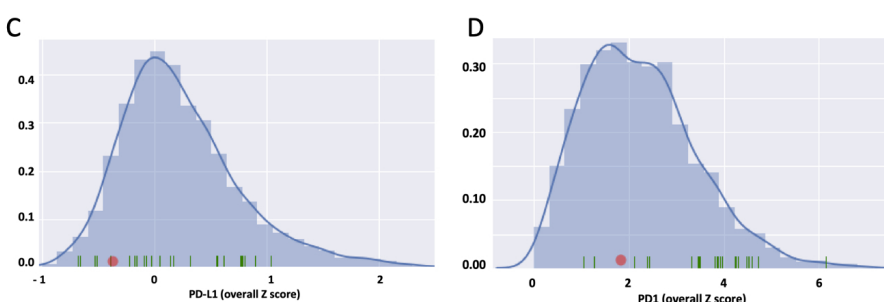

G



J



Figure 3 Expression profile of potential biomarkers of treatment response. (A) For the CNB of the pelvic mass taken before initiation of avelumab treatment, CD8+T cells were lower than average and (B) the CD56dim marker for NK cells was low, particularly compared with other MCCs. (C) PD-L1 was very low as compared with other tumor types and low compared with other MCCs, as was (D) PD-1. (E-G) MGMT, SPARC and TUBB3 were all relatively high. (H) IFN $\gamma$ and (I) TNF $\alpha$ expression was low compared with other tumors and other MCCs. (J) CTLA4 expression was average. The relative expression values for each marker are shown in the Table. CNB, core needle biopsy; IFN $\gamma$, interferon- $\gamma$; MCCs, Merkel cell carcinoma; NK, natural killer; PDL1, programmed death ligand 1.

both the resected tumor and the CNB, but typically positive in SCLC. FLI-1 was positive in the CMB here, and can be positive in SCLC and some MCC. Other positive labeling in MCC typically includes CD56, (negative in the primary and CNB), chromogranin (negative), synaptophysin (weakly positive in both) and neurofilament (not performed). While CD99 positivity is strongly associated with MCPyV and CD99 was positive in the CNB here, the tissue was negative.

\section{Genomic studies}

The patient's tumor specimen was analyzed using the GPS Cancer assay (NantHealth, Culver City, California, USA) comprizing whole exome sequencing and compared with a normal, germline sample collected from peripheral blood; whole transcriptome sequencing (RNA-seq) was also performed. Details of the genomic studies can be viewed in online supplementary results. Forty-four non-synonymous variants, with an estimated exonic mutation rate of 2.1 mutations per megabase of DNA were identified, classifying the tumor as TMB low (figure 2A). No MCPyV expression was detected nor was there a UV signature. As described in Knepper et al, there is a subset of MCC that does not have either the UV signature or MCPyV expression. ${ }^{2}$

\section{NE phenotype}

To predict an NE phenotype, expression levels of a panel of 16 genes associated with NE signaling were determined and compared with a background tumor cohort and an expression quantile generated per each gene. The final NE score is the median quantile across the 16 quantiles. The cut-off for the score for a tumor to be confidently considered NE is 0.8 . Here, the CNB biopsy NE score was 0.74 .

To allow visualization of the tumor character relative to the background cohort, a T-distributed Stochastic Neighbor Embedding plot was generated based on the top 3000 most varying genes. The tumor clustered with sarcomas and some NE tumors, although not with the main NE cluster (figure 2B,C). Thus, some, but not all, evidence supports the NE nature of the tumor.

\section{Gene expression analysis}

The case was compared with a wide range of other cancers and MCG cases from TCGA RNA-Seq FASTQ files. A panel of 109 genes that accurately discriminates between 23 immune cell subpopulations found no elevation of immune cell signatures when compared with other cancer types or to other MCC cases, thus, the tumor could be considered immune 'cold'. Specifically, the CD8+T cell signature was not significantly increased (figure 3A) suggesting limited $\mathrm{T}$ cell infiltration of the tumor microenvironment. Similar analysis predicted the NK population to be low (figure $3 \mathrm{~B}$ ). The CNB did not express PD-L1 (figure 3C) and showed average expression of PD-1 (figure 3D) when compared with other 
tumors. In addition, the expression of O6-MethylguanineDNA-methyltransferase,${ }^{24}$ glycoprotein secreted protein acidic and rich in cysteine/osteonectin (SPARC), and $\beta$-III tubulin (TUBB3) were relatively high in comparison to the cohorts used for analysis (figure $3 \mathrm{E}-\mathrm{G}$, respectively). Abraxane is able to bind to SPARC, leading to its accumulation in the tumor environment and enhanced activity. ${ }^{25}$ Higher TUBB3 expression, in contrast, has been correlated with poorer Abraxane response. ${ }^{26}$

\section{DISCUSSION}

Advanced, metastatic MCC has been historically very difficult to treat, and immune checkpoint refractory patients have almost no viable therapeutic options. Our case of a CR with avelumab, N-803 and Abraxane after initial checkpoint inhibitor failure is unique because of the clinical result and the absence of conventional immunotherapy response-related biomarkers. Molecular analysis revealed features consistent with average immune cell infiltration, low TMB (although just above the cut-off for TMB of 2.0 for efficacy as described in Georges et $a l,{ }^{27}$ MSS and negative status for both PD-L1 and CTLA-4; yet the patient still achieved a CR suggesting the mechanisms by which N-804 and Abraxane work in concert with avelumab to amplify the antitumor response were critical to effective therapy.
Further, as described in Knepper et al, $22 \%$ of MCC do not have either the UV signature or MCPyV expressionas exemplified by the CNB here-and receive less benefit from checkpoint monotherapy than UV-driven MCC. ${ }^{2}$ This also points to the rationale for use of combined therapy here.

\section{CONCLUSION}

As illustrated in figure 4 and based on the known immunobiologies of the agents used, we propose the mechanism of synergy for avelumab, Abraxane, and N-803 is through release of TAAs by Abraxane cytotoxicity that attract a response from $\mathrm{T}$ cells freed from checkpoint suppression by avelumab. Other anti-PD-L1 therapies have shown greater efficacy when combined with Abraxane, for example, atezolizumab, ${ }^{20}$ which is now approved for TNBC; and pembrolizumab plus Abraxane (or paclitaxel plus carboplatin) for NSCLC. ${ }^{28}$ Here, this synergy was enhanced by N-803-mediated stimulation of $\mathrm{T}$ effector cells, ${ }^{10}$ suppression of myeloid-derived suppressor cells and thus $\mathrm{T}$ regulatory cells, $\mathrm{T}$ cell-independent cytotoxicity via NK cell upregulation and possibly PD-L1 upregulation. Further elucidation of the exact mechanism of efficacy for this therapeutic combination awaits a carefully designed clinical trial, given that for this individual

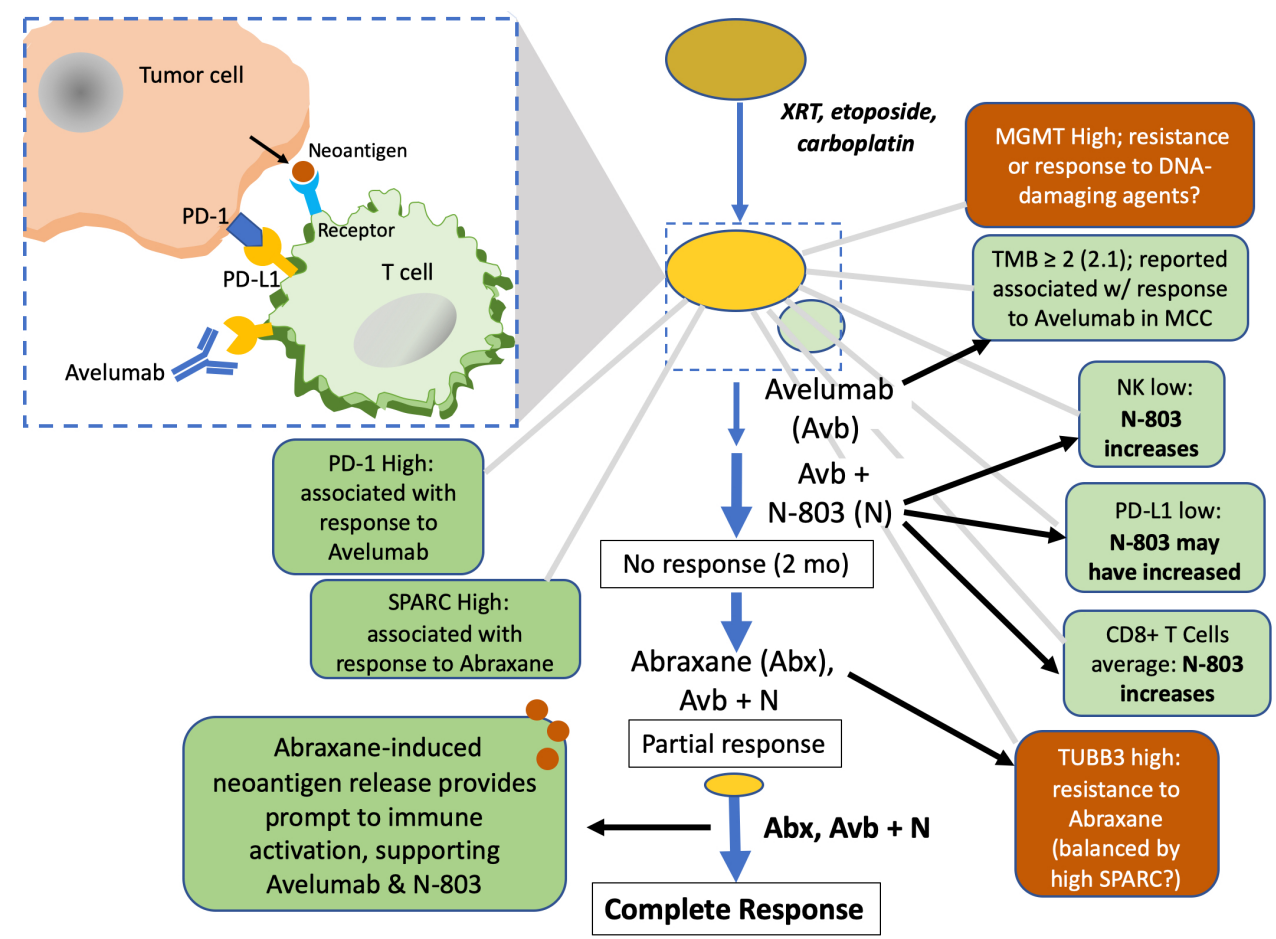

Figure 4 Path to complete response. Initial treatment with DNA-damaging agents led to stable disease, followed by progression. Tissue from the CNB showed a low TMB of $\geq 2$, associated with response to avelumab (in combination with other factors) in MCC; nonetheless, avelumab (anti-PD-L1) monotherapy did not result in a notable response. IL-15 superagonist $\mathrm{N}-803$ likely increased both CD8+Tand NK cells and may have increased PD-L1 expression, but its addition for treatment of this relatively cold tumor was not adequate to elicit an effective response in a 2-month time frame. Abraxane was added, and may have 'primed' the immune system - now unfettered by checkpoint suppression and enhanced by N-803-by release of TAAs neoantigens from apoptotic tumor cells that resulted in T cell infiltration and a CR. CNB, core needle biopsy; CR, complete response; IL-15, interleukin 15; MCCs, Merkel cell carcinoma; MGMT, O6-Methylguanine-DNA-methyltransferase; NK, natural killer; PD-L1, programmed death ligand 1; TAA, tumor cell-associated antigens; TMB, tumor mutational burden. 
patient there was no longitudinal collection of blood and tumor samples before and after each treatment that could undergo analyzes for immune correlates of response; such collection is not part of standard of care. Nonetheless, this case study suggests a promising new treatment modality for similar patients.

Contributors LD co-wrote manuscript and provided records; CN was the patient's physician and provided records; AN, CS and YN performed molecular analysis; PS wrote the manuscript; SBR conceptualized and edited the manuscript.

Funding Chan Soon-Shiong Institute for Medicine.

Competing interests None declared.

Patient consent for publication Obtained.

Provenance and peer review Not commissioned; externally peer reviewed.

Open access This is an open access article distributed in accordance with the Creative Commons Attribution Non Commercial (CC BY-NC 4.0) license, which permits others to distribute, remix, adapt, build upon this work non-commercially, and license their derivative works on different terms, provided the original work is properly cited, appropriate credit is given, any changes made indicated, and the use is non-commercial. See http://creativecommons.org/licenses/by-nc/4.0/.

\section{REFERENCES}

1 Liu W, MacDonald M, You J. Merkel cell polyomavirus infection and Merkel cell carcinoma. Curr Opin Virol 2016;20:20-7.

2 Knepper TC, Montesion M, Russell JS, et al. The genomic landscape of Merkel cell carcinoma and Clinicogenomic biomarkers of response to immune checkpoint inhibitor therapy. Clin Cancer Res 2019;25:5961-71.

3 Goh G, Walradt T, Markarov V, et al. Mutational landscape of MCPyV-positive and MCPyV-negative Merkel cell carcinomas with implications for immunotherapy. Oncotarget 2016;7:3403-15.

4 Garcia-Carbonero R, Marquez-Rodas I, de la Cruz-Merino L, et al. Recent therapeutic advances and change in treatment paradigm of patients with Merkel cell carcinoma. Oncologist 2019;24:1375-83.

5 Samimi M. Immune checkpoint inhibitors and beyond: an overview of immune-based therapies in Merkel cell carcinoma. Am J Clin Dermatol 2019;20:391-407.

6 Kaufman HL, Russell JS, Hamid O, et al. Updated efficacy of avelumab in patients with previously treated metastatic Merkel cell carcinoma after $\geq 1$ year of follow-up: JAVELIN Merkel 200, a phase 2 clinical trial. J Immunother Cancer 2018;6:7.

7 D'Angelo SP, Russell J, Lebbé C, et al. Efficacy and safety of first-line Avelumab treatment in patients with stage IV metastatic Merkel cell carcinoma: a Preplanned interim analysis of a clinical trial. JAMA Oncol 2018;4:e180077.

8 Gaiser MR, Bongiorno M, Brownell I. Pd-L1 inhibition with avelumab for metastatic Merkel cell carcinoma. Expert Rev Clin Pharmacol 2018;11:345-59.

9 Xu W, Jones M, Liu B, et al. Efficacy and mechanism-of-action of a novel superagonist interleukin-15: interleukin-15 receptor $\alpha \mathrm{Su} / \mathrm{Fc}$ fusion complex in syngeneic murine models of multiple myeloma. Cancer Res 2013;73:3075-86.

10 Wong HC, Jeng EK, Rhode PR. The IL-15-based superagonist ALT-803 promotes the antigen-independent conversion of memory
$\mathrm{CD}^{+} \mathrm{T}$ cells into innate-like effector cells with antitumor activity. Oncoimmunology 2013;2:e26442.

11 Kim PS, Kwilas AR, Xu W, et al. II-15 superagonist/IL-15RoSushiFc fusion complex (IL-15SA/IL-15R $\alpha$ Su-Fc; ALT-803) markedly enhances specific subpopulations of NK and memory CD8+ T cells, and mediates potent anti-tumor activity against murine breast and colon carcinomas. Oncotarget 2016;7:16130-45.

12 Mathios D, Park C-K, Marcus WD, et al. Therapeutic administration of IL-15 superagonist complex ALT-803 leads to long-term survival and durable antitumor immune response in a murine glioblastoma model. Int J Cancer 2016;138:187-94.

13 Rosario M, Liu B, Kong L, et al. The IL-15 superagonist ALT-803 enhances NK cell ADCC and in vivo clearance of B cell lymphomas directed by an anti-CD20 monoclonal antibody. Blood 2014;124:807.

14 Kinter AL, Godbout EJ, McNally JP, et al. The common gammachain cytokines IL-2, IL-7, IL-15, and IL-21 induce the expression of programmed death-1 and its ligands. J Immunol 2008;181:6738-46.

15 Knudson KM, Hicks KC, Alter S, et al. Mechanisms involved in IL-15 superagonist enhancement of anti-PD-L1 therapy. J Immunother Cancer 2019;7:82

16 Wrangle JM, Velcheti V, Patel MR, et al. ALT-803, an IL-15 superagonist, in combination with nivolumab in patients with metastatic non-small cell lung cancer: a non-randomised, openlabel, phase 1B trial. Lancet Oncol 2018;19:694-704.

17 Tan H, Hu J, Liu S. Efficacy and safety of nanoparticle albuminbound paclitaxel in non-small cell lung cancer: a systematic review and meta-analysis. Artif Cells Nanomed Biotechnol 2019;47:268-77.

18 Untch M, Jackisch C, Schneeweiss A, et al. Nab-Paclitaxel versus solvent-based paclitaxel in neoadjuvant chemotherapy for early breast cancer (GeparSepto-GBG 69): a randomised, phase 3 trial. Lancet Oncol 2016;17:345-56.

19 Miyasaka Y, Ohtsuka T, Kimura R, et al. Neoadjuvant chemotherapy with gemcitabine plus nab-paclitaxel for borderline resectable pancreatic cancer potentially improves survival and facilitates surgery. Ann Surg Oncol 2019;26:1528-34.

20 Schmid P, Adams S, Rugo HS, et al. Atezolizumab and nabpaclitaxel in advanced triple-negative breast cancer. N Engl J Med 2018;379:2108-21

21 Addeo A, Banna GL, Metro G, et al. Chemotherapy in combination with immune checkpoint inhibitors for the first-line treatment of patients with advanced non-small cell lung cancer: a systematic review and Literature-Based meta-analysis. Front Oncol 2019;9:264-5.

22 Chan OT, Yang LX. The immunological effects of taxanes. Cancer Immunol Immun 2000;49:181-5.

23 Landreneau JP, Shurin MR, Agassandian MV, et al. Immunological mechanisms of low and ultra-low dose cancer chemotherapy. Cancer Microenviron 2015;8:57-64.

24 Sharma S, Salehi F, Scheithauer BW, et al. Role of MGMT in tumor development, progression, diagnosis, treatment and prognosis. Anticancer Res 2009;29:3759-68.

25 Komiya K, Nakamura T, Nakashima C, et al. Sparc is a possible predictive marker for albumin-bound paclitaxel in non-small-cell lung cancer. Onco Targets Ther 2016;9:6663-8.

26 Kanakkanthara A, Teesdale-Spittle PH, Miller JH. Cytoskeletal alterations that confer resistance to anti-tubulin chemotherapeutics. Anticancer Agents Med Chem 2013;13:147-58.

27 Georges S, Shah PK, Shapiro I, et al. Integrative molecular analysis of metastatic Merkel cell carcinoma to identify predictive biomarkers of response to avelumab. JCO 2019;37:9569-70.

28 Paz-Ares L, Luft A, Vicente D, et al. Pembrolizumab plus chemotherapy for squamous non-small-cell lung cancer. $N$ Engl $J$ Med 2018;379:2040-51. 